\title{
Extensive entropy from unitary evolution
}

\author{
Yichen Huang (黄溢辰)* \\ Center for Theoretical Physics, Massachusetts Institute of Technology, \\ Cambridge, Massachusetts 02139, $\mathrm{USA}^{\dagger}$
}

April 7, 2021

\begin{abstract}
In quantum many-body systems, a Hamiltonian is called an "extensive entropy generator" if starting from a random product state the entanglement entropy obeys a volume law at long times with overwhelming probability. We prove that (i) any Hamiltonian whose spectrum has non-degenerate gaps is an extensive entropy generator; (ii) in the space of (geometrically) local Hamiltonians, the non-degenerate gap condition is satisfied almost everywhere. Specializing to many-body localized systems, these results imply the observation stated in the title of Bardarson et al. [PRL 109, 017202 (2012)] $]^{1}$
\end{abstract}

\section{Introduction}

Entropy is a fundamental concept in thermodynamics and statistical mechanics. In textbooks and introductory courses, we learned that entropy is an extensive quantity:

Initializing a system in a low entropy state, generically (although not always) the entropy will grow with time and eventually become proportional to the system size.

The main contribution of this paper is to provide a mathematical characterization of this statement in quantum many-body systems. Our rigorous results not only show how extensive entropy emerges from the unitary evolution under a (geometrically) local Hamiltonian, but also demonstrate the genericness of extensive entropy.

Suppose an isolated quantum spin system is initialized in a pure state. Under unitary evolution the system stays in a pure state and hence its entropy is always zero. To observe

\footnotetext{
*yichuang@mit.edu

${ }^{\dagger}$ Work from home in Commerce Township, Michigan 48390, USA, where winter is cold and snowy.

${ }^{1}$ I first met my doctoral advisor Joel E. Moore in December 2011, when he was working with his group members on the project [4. More than nine years later, I was very fortunate to find a proof of the observation stated in the title of Ref. [4]. This reminds me of the sunny summer and rainy (not snowy) winter in Berkeley. I am very grateful to Joel for his guidance and support since we first met.
} 
non-trivial entropy dynamics, we divide the system into two parts $A$ and $B$. Assume without loss of generality that subsystem $A$ is smaller than or equal to half the system size. We view $B$ as a bath of $A$ and consider the entropy of $A$. This entropy is called entanglement entropy, and extensive subsystem entropy is also known as a volume law for entanglement. The most general unentangled state with zero entropy for all subsystems is a random product state, where each spin is chosen independently and uniformly at random on the Bloch sphere.

Our main result is the following. A Hamiltonian is called an "extensive entropy generator" if starting from a random product state the entanglement entropy obeys a volume law at long times with overwhelming probability. We prove that (i) any Hamiltonian whose spectrum has non-degenerate gaps is an extensive entropy generator; (ii) in the space of Hamiltonians with short-range interactions, the non-degenerate gap condition is satisfied almost everywhere.

As a byproduct, we solve a mathematical problem in many-body localization. It is well known that in one-dimensional Anderson localized systems, starting from a random product state the entanglement entropy remains bounded at all times [1]. However, upon adding a generic local perturbation the system becomes many-body localized (MBL), and unbounded growth of entanglement was observed numerically [20, 4, 12] and then explained heuristically [18, 15, 8]. We prove this observation.

\section{Preliminaries}

Throughout this paper, standard asymptotic notations are used extensively. Let $f, g: \mathbb{R}^{+} \rightarrow$ $\mathbb{R}^{+}$be two functions. One writes $f(x)=O(g(x))$ if and only if there exist constants $M, x_{0}>0$ such that $f(x) \leq M g(x)$ for all $x>x_{0} ; f(x)=\Omega(g(x))$ if and only if there exist constants $M, x_{0}>0$ such that $f(x) \geq M g(x)$ for all $x>x_{0}$.

Definition 1 (entanglement entropy). The entanglement entropy of a bipartite pure state $\rho_{A B}$ is defined as the von Neumann entropy

$$
S\left(\rho_{A}\right):=-\operatorname{tr}\left(\rho_{A} \ln \rho_{A}\right)
$$

of the reduced density matrix $\rho_{A}=\operatorname{tr}_{B} \rho_{A B}$.

Consider a system of $N$ spins or qudits with local dimension $d$ so that the dimension of the total Hilbert space is $d^{N}$.

Definition 2 (Haar-random product state). Let $|\Psi\rangle=\bigotimes_{j=1}^{N}\left|\Psi_{j}\right\rangle$ be a Haar-random product state, where each $\left|\Psi_{j}\right\rangle$ is chosen independently and uniformly at random with respect to the Haar measure.

Definition 3 (non-degenerate spectrum). The spectrum of a Hamiltonian is non-degenerate if all eigenvalues are distinct.

Definition 4 (non-degenerate gaps). The spectrum $\left\{E_{j}\right\}$ of a Hamiltonian has non-degenerate gaps if the differences $\left\{E_{j}-E_{k}\right\}_{j \neq k}$ are all distinct, i.e., for any $j \neq k$,

$$
E_{j}-E_{k}=E_{j^{\prime}}-E_{k^{\prime}} \Longrightarrow\left(j=j^{\prime}\right) \text { and }\left(k=k^{\prime}\right) \text {. }
$$

By definition, the non-degenerate gap condition implies that the spectrum is non-degenerate. 


\section{Results}

Let $m, n$ be positive integers such that $m n$ is a multiple of the system size $N$. Let $A_{1}, A_{2}, \ldots, A_{m}$ be $m$ possibly overlapping subsystems, each of which consists of exactly $n \leq N / 2$ spins. Suppose that each spin in the system is in exactly $m n / N$ out of these $m$ subsystems. For each $j$, let $\bar{A}_{j}$ be the complement of $A_{j}$ so that $A_{j} \otimes \bar{A}_{j}$ defines a bipartition of the system.

Theorem 1. Initialize the system in a Haar-random product state $|\Psi\rangle$ (Definition 2). Let

$$
\rho_{A_{j}}(t):=\operatorname{tr}_{\bar{A}_{j}} \rho(t), \quad \rho(t):=e^{-i H t}|\Psi\rangle\langle\Psi| e^{i H t}
$$

be the reduced density matrix of subsystem $A_{j}$ at time $t$. We consider the scenario that

$$
n \leq \frac{(1-\epsilon) N \ln \frac{d+1}{2}}{2 \ln d}
$$

where $\epsilon>0$ is an arbitrarily small constant. For any Hamiltonian $H$ whose spectrum has non-degenerate gaps (Definition 4),

$$
\operatorname{Pr}_{\Psi}\left(\operatorname{Pr}_{t \in \mathbb{R}}\left(\frac{1}{m} \sum_{j=1}^{m} S\left(\rho_{A_{j}}(t)\right) \geq(1-\epsilon) n \sum_{k=2}^{d} \frac{1}{k}\right)=1-e^{-\Omega(N)}\right)=1-e^{-\Omega(N)} .
$$

There is no underlying lattice structure in the statement of Theorem 1. From now on we focus on quantum lattice systems, which are of particular interest. Without loss of generality, we consider a chain of $N$ spins. It is straightforward to extend Corollary 1 below to higher spatial dimensions.

Let $A$ be a contiguous region of $n$ spins, and $\bar{A}$ be the rest of the system. Let $\mathbb{E}_{|A|=n}$ denote averaging over all contiguous subsystems of size $n$. With periodic boundary conditions, there are $N$ such subsystems.

Corollary 1. Initialize the system in a Haar-random product state $|\Psi\rangle$. Let

$$
\rho_{A}(t):=\operatorname{tr}_{\bar{A}} \rho(t), \quad \rho(t):=e^{-i H t}|\Psi\rangle\langle\Psi| e^{i H t}
$$

be the reduced density matrix of subsystem $A$ at time $t$. We consider the scenario that

$$
\frac{(1-\epsilon) N \ln \frac{d+1}{2}}{2 \ln d}<n \leq N / 2
$$

where $\epsilon>0$ is an arbitrarily small constant. For any (not necessarily local) Hamiltonian $H$ whose spectrum has non-degenerate gaps,

$$
\operatorname{Pr}_{\Psi}\left(\operatorname{Pr}_{t \in \mathbb{R}}\left(\underset{|A|=n}{\mathbb{E}} S\left(\rho_{A}(t)\right) \geq\left(\frac{1}{2}-\epsilon\right) \frac{N \ln \frac{d+1}{2}}{\ln d} \sum_{k=2}^{d} \frac{1}{k}\right)=1-e^{-\Omega(N)}\right)=1-e^{-\Omega(N)} .
$$

Proof. The weak monotonicity [10] of the von Neumann entropy implies that

$$
\underset{|A|=n}{\mathbb{E}} S\left(\rho_{A}(t)\right) \leq \underset{|A|=n+1}{\mathbb{E}} S\left(\rho_{A}(t)\right)
$$

for any $n<N / 2$. Corollary 1 follows from this inequality and Theorem 1 . 
To demonstrate the genericness of non-degenerate gaps, we define ensembles of Hamiltonians with nearest-neighbor interactions in a chain of $N$ spin-1/2's or qubits $(d=2)$. We prove that in each ensemble, the set of Hamiltonians whose spectrum has degenerate gaps is of measure zero (Theorem 2). Similar results can be proved in a similar way for other types of systems including qudit systems with short-range interactions in higher spatial dimensions or even with non-local interactions (Appendix C).

The non-degenerate gap condition (2) was assumed in many previous works [13, 11, 16, 17, 14, 6, 19] on the equilibration of quantum systems. Both Theorem 1 and the results of these works apply to almost every Hamiltonian in each ensemble we define.

Let

$$
\hat{\sigma}_{j}^{x}=\left(\begin{array}{ll}
0 & 1 \\
1 & 0
\end{array}\right), \quad \hat{\sigma}_{j}^{y}=\left(\begin{array}{cc}
0 & -i \\
i & 0
\end{array}\right), \quad \hat{\sigma}_{j}^{z}=\left(\begin{array}{cc}
1 & 0 \\
0 & -1
\end{array}\right)
$$

be the Pauli matrices for the spin- $1 / 2$ at position $j$, and

$$
\begin{aligned}
& J:=\left(\left.J_{k}\right|_{k \in\{x, y, z\}},\left.J_{k l}\right|_{k, l \in\{x, y, z\}}\right)=\left(J_{x}, J_{y}, J_{z}, J_{x x}, J_{x y}, J_{x z}, J_{y x}, J_{y y}, J_{y z}, J_{z x}, J_{z y}, J_{z z}\right), \\
& \alpha:=\left(\left.\alpha_{j}^{k}\right|_{1 \leq j \leq N} ^{k \in\{x, y, z\}},\left.\alpha_{j}^{k l}\right|_{1 \leq j \leq N-1} ^{k, l \in\{x, y, z\}}\right), \quad R:=(0,1] \times[0,1] \times(0,1] \times[0,1]^{\times 8} \times(0,1] \subset \mathbb{R}^{12} .
\end{aligned}
$$

Each particular $J$ defines an ensemble of Hamiltonians

$$
\begin{aligned}
& H_{J}:=\left\{H_{J}(\alpha): \alpha \in[-1,1]^{\times(12 N-9)}\right\} \\
& H_{J}(\alpha):=\sum_{j=1}^{N} \sum_{k \in\{x, y, z\}} J_{k} \alpha_{j}^{k} \hat{\sigma}_{j}^{k}+\sum_{j=1}^{N-1} \sum_{k, l \in\{x, y, z\}} J_{k l} \alpha_{j}^{k l} \hat{\sigma}_{j}^{k} \hat{\sigma}_{j+1}^{l} .
\end{aligned}
$$

Note that $J \in R$ implies $J_{x}, J_{z}, J_{z z}>0$. This rules out the possibility that $H_{J}$ is an ensemble of free-fermion Hamiltonians.

Theorem 2. For any $J \in R$, the set of all $\alpha$ such that the spectrum of $H_{J}(\alpha)$ has degenerate gaps is of measure zero.

It is an open problem to prove an analogue of this theorem for translationally invariant systems. Progress in this direction was made in Ref. [7].

\section{Many-body localization}

$H_{J}$ is MBL for some $J \in R$. In particular, $H_{J}$ reduces to the Imbrie model [9] when

$$
J=(\lambda, 0,1,0,0,0,0,0,0,0,0,1)
$$

with small $\lambda>0$.

Other models of MBL can be considered. For example, let

$$
h:=\left(h_{x}, h_{z}, h_{z z}\right), \quad \gamma:=\left(\left.\gamma_{j}^{k}\right|_{1 \leq j \leq N} ^{k \in\{x, z\}},\left.\gamma_{j}^{z z}\right|_{1 \leq j \leq N-1}\right) .
$$

Each particular $h$ defines an ensemble of Hamiltonians

$$
\begin{aligned}
& H_{h}^{X X Z}:=\left\{H_{h}^{X X Z}(\gamma): \gamma \in[-1,1]^{\times(3 N-1)}\right\} \\
& H_{h}^{X X Z}(\gamma)=\sum_{j=1}^{N}\left(h_{x} \gamma_{j}^{x} \hat{\sigma}_{j}^{x}+h_{z} \gamma_{j}^{z} \hat{\sigma}_{j}^{z}\right)+\sum_{j=1}^{N-1}\left(\hat{\sigma}_{j}^{x} \hat{\sigma}_{j+1}^{x}+\hat{\sigma}_{j}^{y} \hat{\sigma}_{j+1}^{y}+h_{z z} \gamma_{j}^{z z} \hat{\sigma}_{j}^{z} \hat{\sigma}_{j+1}^{z}\right) .
\end{aligned}
$$

Note that $H_{h}^{X X Z}$ is a perturbed random-field $X X$ chain if $h_{x}, h_{z z}>0$ are small. 
Corollary 2. For any $h \in(0,+\infty)^{\times 3}$, the set of all $\gamma$ such that the spectrum of $H_{h}^{X X Z}(\gamma)$ has degenerate gaps is of measure zero.

The result stated in the last paragraph of the introduction follows by combining Theorem 1 and Corollary 2 .

Finally, we prove that the volume-law coefficient in Eq. (5) is tight in a particular MBL system. Consider a chain of $N$ qudits labeled by $1,2, \ldots, N$. Let $\hat{S}_{j}^{z}$ be the $z$ component of the spin operator at position $j$. Let $\left(h_{1}, h_{2}, \ldots, h_{N}\right) \in \mathbb{R}^{N}$ be such that the spectrum of

$$
H^{\mathrm{loc}}:=\sum_{j=1}^{N} h_{j} \hat{S}_{j}^{z}
$$

is non-degenerate. Let $H^{\mathrm{mbl}}:=H^{\mathrm{loc}}+\Delta H$, where $\Delta H$ is an infinitesimal random local perturbation. Appendix C.3 proves that the spectrum of $H^{\mathrm{mbl}}$ almost surely has non-degenerate gaps. Let $A \subset\{1,2, \ldots, N\}$ so that $A \sqcup \bar{A}$ defines a bipartition of the system.

Theorem 3. Initialize the system in a Haar-random product state $|\Psi\rangle$. Let

$$
\rho_{A}(t):=\operatorname{tr}_{\bar{A}} \rho(t), \quad \rho(t):=e^{-i H^{\mathrm{mbl}} t}|\Psi\rangle\langle\Psi| e^{i H^{\mathrm{mbl}} t}
$$

be the reduced density matrix of subsystem $A$ at time $t$. For any $A$,

$$
\underset{\Psi}{\mathbb{E}} \lim _{\tau \rightarrow+\infty} \frac{1}{\tau} \int_{0}^{\tau} S\left(\rho_{A}(t)\right) \mathrm{d} t \leq|A| \sum_{k=2}^{d} \frac{1}{k} .
$$

\section{Acknowledgments}

I would like to thank my postdoctoral advisor Aram W. Harrow for comments on this paper and for collaboration on a related project [7]. This work was supported by NSF grant PHY1818914 and a Samsung Advanced Institute of Technology Global Research Partnership.

\section{A Proof of Theorem 1}

Lemma 1. Let $|0\rangle$ be a particular state in $\mathbb{C}^{d}$. For a Haar-random state $\left|\Psi^{\prime}\right\rangle$,

$$
M_{\alpha}:=\underset{\Psi^{\prime}}{\mathbb{E}}\left|\left\langle 0 \mid \Psi^{\prime}\right\rangle\right|^{2 \alpha}=\prod_{j=1}^{d-1} \frac{j}{j+\alpha} .
$$

Proof. Calculating a tedious multivariable integral, the probability density function of the random variable $X:=\left|\left\langle 0 \mid \Psi^{\prime}\right\rangle\right|^{2}$ is

$$
f(x)=(d-1)(1-x)^{d-2}, \quad x \in[0,1] .
$$

Therefore,

$$
M_{\alpha}=\int_{0}^{1} x^{\alpha} f(x) \mathrm{d} x=\prod_{j=1}^{d-1} \frac{j}{j+\alpha} .
$$


Lemma 2. Let $|\phi\rangle$ be an arbitrary state in $\left(\mathbb{C}^{d}\right)^{\otimes N}$. For a Haar-random product state $|\Psi\rangle$ (Definition 2) and any $\alpha \geq 1$,

$$
\underset{\Psi}{\mathbb{E}}|\langle\phi \mid \Psi\rangle|^{2 \alpha} \leq M_{\alpha}^{N}
$$

Proof. Let $A \otimes \bar{A}$ be a bipartition of the system, where subsystem $A$ consists of a single spin. Let $\left\{|j\rangle_{A}\right\}_{j=0}^{d-1}$ be the computational basis of subsystem $A$ so that

$$
|\phi\rangle=\sum_{j=0}^{d-1} c_{j}|j\rangle_{A} \otimes\left|\phi_{j}\right\rangle_{\bar{A}}, \quad \sum_{j=0}^{d-1}\left|c_{j}\right|^{2}=1
$$

Let $|\Psi\rangle=\left|\Psi_{A}\right\rangle \otimes\left|\Psi_{\bar{A}}\right\rangle$, where $\left|\Psi_{A}\right\rangle$ is a Haar-random state in $\mathbb{C}^{d}$ and $\left|\Psi_{\bar{A}}\right\rangle$ is a Haar-random product state in $\left(\mathbb{C}^{d}\right)^{\otimes(N-1)}$. It is easy to see that for any fixed $\left|\Psi_{\bar{A}}\right\rangle$,

$$
\underset{\Psi_{A}}{\mathbb{E}}|\langle\phi \mid \Psi\rangle|^{2 \alpha}=M_{\alpha}\left(\sum_{j=0}^{d-1}\left|c_{j}\right|^{2}\left|\left\langle\phi_{j} \mid \Psi_{\bar{A}}\right\rangle\right|^{2}\right)^{\alpha} \leq M_{\alpha} \sum_{j=0}^{d-1}\left|c_{j}\right|^{2}\left|\left\langle\phi_{j} \mid \Psi_{\bar{A}}\right\rangle\right|^{2 \alpha}
$$

Hence,

$$
\underset{\Psi}{\mathbb{E}}|\langle\phi \mid \Psi\rangle|^{2 \alpha}=\underset{\Psi_{\bar{A}}}{\mathbb{E}} \underset{\Psi_{A}}{\mathbb{E}}|\langle\phi \mid \Psi\rangle|^{2 \alpha} \leq M_{\alpha} \sum_{j=0}^{d-1}\left|c_{j}\right|^{2} \underset{\Psi_{\bar{A}}}{\mathbb{E}}\left|\left\langle\phi_{j} \mid \Psi_{\bar{A}}\right\rangle\right|^{2 \alpha} \leq M_{\alpha} \max _{\phi^{\prime}} \underset{\Psi_{\bar{A}}}{\mathbb{E}}\left|\left\langle\phi^{\prime} \mid \Psi_{\bar{A}}\right\rangle\right|^{2 \alpha},
$$

where $\left|\phi^{\prime}\right\rangle$ is a state in $\left(\mathbb{C}^{d}\right)^{\otimes(N-1)}$. We obtain 25 by iteratively applying 28 .

Lemma 3. Let $\left\{\left|b_{1}\right\rangle,\left|b_{2}\right\rangle, \ldots,\left|b_{d^{N}}\right\rangle\right\}$ be an arbitrary orthonormal basis of the Hilbert space $\left(\mathbb{C}^{d}\right)^{\otimes N}$. For a Haar-random product state $|\Psi\rangle$ and any $\alpha \geq 1$,

$$
\underset{\Psi}{\mathbb{E}} \sum_{j=1}^{d^{N}}\left|\left\langle b_{j} \mid \Psi\right\rangle\right|^{2 \alpha} \leq\left(M_{\alpha} d\right)^{N}
$$

Furthermore, for an arbitrarily small constant $\varepsilon>0$,

$$
\operatorname{Pr}_{\Psi}\left(\sum_{j=1}^{d^{N}}\left|\left\langle b_{j} \mid \Psi\right\rangle\right|^{2 \alpha} \leq\left(M_{\alpha} d\right)^{(1-\varepsilon) N}\right)=1-e^{-\Omega(N)}
$$

Proof. The probabilistic bound (30) follows from Markov's inequality.

For $\alpha=2$, Lemma 3 reduces to Lemma 5 in Ref. [7].

Let $\{|j\rangle\}_{j=1}^{d^{N}}$ be a complete set of eigenstates of $H$. Assuming that the spectrum of $H$ is non-degenerate, the energy basis $\{|j\rangle\}$ is unambiguously defined. The effective dimension of a state $|\psi\rangle$ is defined as

$$
1 / D_{\psi}^{\mathrm{eff}}=\sum_{j=1}^{d^{N}}|\langle j \mid \psi\rangle|^{4} .
$$


Lemma 4. Initialize the system in a pure state $|\psi\rangle$. Let

$$
\sigma:=\lim _{\tau \rightarrow+\infty} \frac{1}{\tau} \int_{0}^{\tau} \rho(t) \mathrm{d} t, \quad \rho(t):=e^{-i H t}|\psi\rangle\langle\psi| e^{i H t}
$$

be the infinite time average and $\sigma_{A_{j}}:=\operatorname{tr}_{\bar{A}_{j}} \sigma$ be the reduced density matrix of subsystem $A_{j}$. For any Hamiltonian $H$ with non-degenerate spectrum and any $\alpha>1$,

$$
\frac{1}{m} \sum_{j=1}^{m} S\left(\sigma_{A_{j}}\right) \geq \frac{n}{N(1-\alpha)} \ln \sum_{j=1}^{d^{N}}|\langle j \mid \psi\rangle|^{2 \alpha}
$$

Proof. Expanding $|\psi\rangle$ in the energy basis, it is easy to see that

$$
\sigma=\sum_{j=1}^{d^{N}} p_{j}|j\rangle\left\langle\left. j\left|, \quad p_{j}:=\right|\langle j \mid \psi\rangle\right|^{2}\right.
$$

is the so-called diagonal ensemble. Using the strong subadditivity [10] of the von Neumann entropy and the monotonicity of the Rényi entropy,

$$
\frac{1}{m} \sum_{j=1}^{m} S\left(\sigma_{A_{j}}\right) \geq \frac{n}{N} S(\sigma)=-\frac{n}{N} \sum_{j=1}^{d^{N}} p_{j} \ln p_{j} \geq \frac{n}{N(1-\alpha)} \ln \sum_{j=1}^{d^{N}} p_{j}^{\alpha}, \quad \forall \alpha>1 .
$$

Let $\|X\|_{1}:=\operatorname{tr} \sqrt{X^{\dagger} X}$ denote the trace norm.

Lemma 5 ([11, 16]). Using the notation of Lemma 4, for any Hamiltonian $H$ whose spectrum has non-degenerate gaps,

$$
\lim _{\tau \rightarrow+\infty} \frac{1}{\tau} \int_{0}^{\tau}\left\|\rho_{A_{j}}(t)-\sigma_{A_{j}}\right\|_{1} \mathrm{~d} t \leq d^{n} / \sqrt{D_{\psi}^{\mathrm{eff}}}
$$

Lemma 6 (continuity of the von Neumann entropy [5, 3]). Let $T:=\|\rho-\sigma\|_{1} / 2$ be the trace distance between two density matrices $\rho, \sigma$ on the Hilbert space $\mathbb{C}^{D}$. Then,

$$
|S(\rho)-S(\sigma)| \leq T \ln (D-1)-T \ln T-(1-T) \ln (1-T)
$$

Since by definition $0 \leq T \leq 1$, the right-hand side of this inequality is well defined.

We are ready to prove Theorem 1 . Lemmas 3 and 4 imply that

$$
\begin{aligned}
& \operatorname{Pr}_{\Psi}\left(D_{\Psi}^{\mathrm{eff}} \geq\left(\frac{d+1}{2}\right)^{(1-\epsilon / 2) N}\right)=1-e^{-\Omega(N)} \\
& \underset{\Psi}{\operatorname{Pr}}\left(\frac{1}{m} \sum_{j=1}^{m} S\left(\sigma_{A_{j}}\right) \geq \frac{(1-\epsilon / 3) n}{\alpha-1} \sum_{k=2}^{d} \ln \left(1+\frac{\alpha-1}{k}\right)\right)=1-e^{-\Omega(N)}, \quad \forall \alpha>1 .
\end{aligned}
$$


Therefore, it suffices to prove that

$$
\operatorname{Pr}_{t \in \mathbb{R}}\left(\frac{1}{m} \sum_{j=1}^{m} S\left(\rho_{A_{j}}(t)\right) \geq(1-\epsilon) n \sum_{k=2}^{d} \frac{1}{k}\right)=1-e^{-\Omega(N)}
$$

assuming that

$$
\begin{aligned}
& D_{\Psi}^{\mathrm{eff}} \geq\left(\frac{d+1}{2}\right)^{(1-\epsilon / 2) N}, \\
& \frac{1}{m} \sum_{j=1}^{m} S\left(\sigma_{A_{j}}\right) \geq(1-2 \epsilon / 3) n \sum_{k=2}^{d} \frac{1}{k}
\end{aligned}
$$

(4), (36), and (41) imply that

$$
\lim _{\tau \rightarrow+\infty} \frac{1}{\tau} \int_{0}^{\tau} \frac{1}{m} \sum_{j=1}^{m}\left\|\rho_{A_{j}}(t)-\sigma_{A_{j}}\right\|_{1} \mathrm{~d} t \leq d^{n} / \sqrt{D_{\Psi}^{\mathrm{eff}}} \leq\left(\frac{2}{d+1}\right)^{\epsilon N / 4}=e^{-\Omega(N)}
$$

Markov's inequality implies that

$$
\operatorname{Pr}_{t \in \mathbb{R}}\left(\frac{1}{m} \sum_{j=1}^{m}\left\|\rho_{A_{j}}(t)-\sigma_{A_{j}}\right\|_{1}=e^{-\Omega(N)}\right)=1-e^{-\Omega(N)} .
$$

Due to the continuity of the von Neumann entropy (Lemma 6),

$$
\frac{1}{m} \sum_{j=1}^{m}\left\|\rho_{A_{j}}(t)-\sigma_{A_{j}}\right\|_{1}=e^{-\Omega(N)} \Longrightarrow \frac{1}{m} \sum_{j=1}^{m}\left|S\left(\rho_{A_{j}}(t)\right)-S\left(\sigma_{A_{j}}\right)\right|=e^{-\Omega(N)} \text {. }
$$

Equation (40) follows from 42, 44, and 45.

\section{B Proof of Theorem 2}

We begin by following the proof of Lemma 8 in Ref. [7]. Let $\left\{E_{j}\right\}_{j=1}^{2^{N}}$ with be the eigenvalues of $H_{J}(\alpha)$ and

$$
G_{J}(\alpha):=\prod_{\left(\left(j \neq j^{\prime}\right) \vee\left(k \neq k^{\prime}\right)\right) \wedge\left(\left(j \neq k^{\prime}\right) \vee\left(k \neq j^{\prime}\right)\right)}\left(E_{j}+E_{k}-E_{j^{\prime}}-E_{k^{\prime}}\right)
$$

so that $G_{J}(\alpha)=0$ if and only if the spectrum of $H_{J}(\alpha)$ has degenerate gaps (Definition 4 ). It is easy to see that $G_{J}(\alpha)$ is a symmetric polynomial in $E_{1}, E_{2}, \ldots, E_{2^{N}}$. The fundamental theorem of symmetric polynomials implies that $G_{J}(\alpha)$ can be expressed as a polynomial in $F_{1}, F_{2}, \ldots$, where

$$
F_{k}:=\sum_{j=1}^{2^{N}} E_{j}^{k}=\operatorname{tr}\left(H_{J}^{k}(\alpha)\right)
$$


Expanding $H_{J}^{k}(\alpha)$ in the Pauli basis and taking the trace, we see that $F_{k}$ and hence $G_{J}(\alpha)$ : $[-1,1]^{\times(12 N-9)} \rightarrow \mathbb{R}$ are polynomials in the entries of $\alpha$. Since the zeros of a multivariable polynomial are of measure zero unless the polynomial is identically zero, it suffices to find a particular $\alpha \in \mathbb{R}^{12 N-9}$ such that the spectrum of $H_{J}(\alpha)$ has non-degenerate gaps. This is done in the following lemma.

Lemma 7. For any positive integer $N$, there exists $\left(h_{1}, J_{1}, h_{2}, J_{2}, \ldots, h_{N-1}, J_{N-1}\right) \in \mathbb{R}^{2(N-1)}$ such that the spectrum of

$$
H_{N}=\hat{\sigma}_{1}^{z}+\sum_{j=1}^{N-1}\left(h_{j} \hat{\sigma}_{j+1}^{x}+J_{j}\left(2+\hat{\sigma}_{j}^{z}\right) \hat{\sigma}_{j+1}^{z}\right)
$$

has non-degenerate gaps.

Proof. We prove by induction on $N$.

Induction hypotheses. We have two induction hypotheses:

1. The spectrum of $H_{N}$ has non-degenerate gaps.

2. $\left\{\left\langle j\left|\hat{\sigma}_{N}^{z}\right| j\right\rangle\right\}$ is a set of pairwise distinct numbers, where $\{|j\rangle\}_{j=1}^{2^{N}}$ are eigenstates of $H_{N}$.

Base case. The base case $N=1$ is trivially true.

Induction step. Assuming the induction hypotheses for $H_{N}$, we prove those for $H_{N+1}$ using perturbation theory. The unperturbed Hamiltonian and the perturbation are

$$
H_{\mathrm{unp}}:=H_{N}+h_{N} \hat{\sigma}_{N+1}^{x}, \quad H_{\mathrm{per}}:=J_{N}\left(2+\hat{\sigma}_{N}^{z}\right) \hat{\sigma}_{N+1}^{z},
$$

respectively. Let $\{|j\rangle\}$ be eigenstates of $H_{N}$ with corresponding energies $\left\{E_{j}\right\}$. Let

$$
\Delta:=\min _{j \neq k}\left|E_{j}-E_{k}\right|, \quad \delta:=\min _{\left(\left(j \neq j^{\prime}\right) \vee\left(k \neq k^{\prime}\right)\right) \wedge\left(\left(j \neq k^{\prime}\right) \vee\left(k \neq j^{\prime}\right)\right)}\left|E_{j}+E_{k}-E_{j^{\prime}}-E_{k^{\prime}}\right|
$$

so that $\delta \leq \Delta$. Let $|\circ\rangle,|\bullet\rangle$ be eigenstates of $\sigma_{N+1}^{x}$ with eigenvalues \pm 1 . The eigenstates of $H_{\text {unp }}$ are $\{|j \circ\rangle,|j \bullet\rangle\}$ with corresponding energies $\left\{E_{j} \pm h_{N}\right\}$. We choose $h_{N}, J_{N}$ such that

$$
0<J_{N} \ll h_{N} \ll \min \left\{\delta, \Delta \min _{j \neq k}\left|\left\langle j\left|\hat{\sigma}_{N}^{z}\right| j\right\rangle-\left\langle k\left|\hat{\sigma}_{N}^{z}\right| k\right\rangle\right|\right\}
$$

The induction hypotheses imply that the most right-hand side of (51) is positive. The condition (51) implies that the energy gap (minimum difference between adjacent eigenvalues) of $H_{\text {unp }}$ is $2 h_{N}$. Let $\left.\left.\{\mid j \circ), \mid j \bullet\right)\right\}$ be eigenstates of $H_{N+1}:=H_{\text {unp }}+H_{\text {per }}$ with corresponding energies $\left\{E_{j \circ}, E_{j \bullet}\right\}$.

Proof of induction hypothesis 1 for $H_{N+1}$. Using second-order non-degenerate perturbation theory,

$$
\begin{aligned}
E_{j \circ} & =E_{j}+h_{N}+\left\langle j \circ\left|H_{\mathrm{per}}\right| j \circ\right\rangle+\sum_{k \neq j} \frac{\left|\left\langle k \circ\left|H_{\mathrm{per}}\right| j \circ\right\rangle\right|^{2}}{E_{j}-E_{k}}+\sum_{k} \frac{\left|\left\langle k \bullet\left|H_{\mathrm{per}}\right| j \circ\right\rangle\right|^{2}}{E_{j}-E_{k}+2 h_{N}}+O\left(J_{N}^{3}\right) \\
& =E_{j}+h_{N}+\frac{J_{N}^{2}\left(2+\left\langle j\left|\hat{\sigma}_{N}^{z}\right| j\right\rangle\right)^{2}}{2 h_{N}}+\sum_{k \neq j} \frac{J_{N}^{2}\left|\left\langle k\left|\hat{\sigma}_{N}^{z}\right| j\right\rangle\right|^{2}}{E_{j}-E_{k}+2 h_{N}}+O\left(J_{N}^{3}\right) \\
& =E_{j}+h_{N}+\frac{J_{N}^{2}\left(2+\left\langle j\left|\hat{\sigma}_{N}^{z}\right| j\right\rangle\right)^{2}}{2 h_{N}}+O\left(J_{N}^{2} / \Delta\right),
\end{aligned}
$$


where we used

$$
\sum_{k \neq j} \frac{\left|\left\langle k\left|\hat{\sigma}_{N}^{z}\right| j\right\rangle\right|^{2}}{\left|E_{j}-E_{k}+2 h_{N}\right|} \leq \sum_{k} \frac{\left|\left\langle k\left|\hat{\sigma}_{N}^{z}\right| j\right\rangle\right|^{2}}{\Delta-2 h_{N}}=\frac{1}{\Delta-2 h_{N}}=O(1 / \Delta) .
$$

Similarly,

$$
E_{j \bullet}=E_{j}-h_{N}-\frac{J_{N}^{2}\left(2+\left\langle j\left|\hat{\sigma}_{N}^{z}\right| j\right\rangle\right)^{2}}{2 h_{N}}+O\left(J_{N}^{2} / \Delta\right) .
$$

Suppose that

$$
E_{j \alpha}+E_{k \beta}=E_{j^{\prime} \alpha^{\prime}}+E_{k^{\prime} \beta^{\prime}}, \quad \alpha, \beta, \alpha^{\prime}, \beta^{\prime} \in\{\circ, \bullet\} .
$$

Assume without loss of generality that $j \leq k$ and $j^{\prime} \leq k^{\prime}$. Since $h_{N} \ll \delta$, we have $j=j^{\prime}$ and $k=k^{\prime}$. Since $J_{N} \ll h_{N}$, it suffices to exclude the possibilities that (1) $j \neq k$ and $\alpha=\beta^{\prime}=0$ and $\beta=\alpha^{\prime}=\bullet$ or $(2) j \neq k$ and $\alpha=\beta^{\prime}=\bullet$ and $\beta=\alpha^{\prime}=0$. In both cases,

$$
\begin{aligned}
\left|J_{N}^{2}\left(2+\left\langle j\left|\hat{\sigma}_{N}^{z}\right| j\right\rangle\right)^{2} / h_{N}-J_{N}^{2}\left(2+\left\langle k\left|\hat{\sigma}_{N}^{z}\right| k\right\rangle\right)^{2} / h_{N}\right| & =O\left(J_{N}^{2} / \Delta\right) \\
& \Longrightarrow\left|\left\langle j\left|\hat{\sigma}_{N}^{z}\right| j\right\rangle-\left\langle k\left|\hat{\sigma}_{N}^{z}\right| k\right\rangle\right|=O\left(h_{N} / \Delta\right) .
\end{aligned}
$$

This is impossible for our choice of $h_{N}(51)$.

Proof of induction hypothesis 2 for $H_{N+1}$. Using first-order non-degenerate perturbation theory,

$$
\begin{aligned}
\mid j \circ)=|j \circ\rangle+\sum_{k \neq j} & \frac{\left\langle k \circ\left|H_{\mathrm{per}}\right| j \circ\right\rangle}{E_{j}-E_{k}}|k \circ\rangle+\sum_{k} \frac{\left\langle k \bullet\left|H_{\mathrm{per}}\right| j \circ\right\rangle}{E_{j}-E_{k}+2 h_{N}}|k \bullet\rangle+O\left(J_{N}^{2}\right) \\
& =|j \circ\rangle+\frac{J_{N}\left(2+\left\langle j\left|\hat{\sigma}_{N}^{z}\right| j\right\rangle\right)}{2 h_{N}}|j \bullet\rangle+\sum_{k \neq j} \frac{J_{N}\left\langle k\left|\hat{\sigma}_{N}^{z}\right| j\right\rangle}{E_{j}-E_{k}+2 h_{N}}|k \bullet\rangle+O\left(J_{N}^{2}\right)
\end{aligned}
$$

so that

$$
\left(j \circ\left|\hat{\sigma}_{N+1}^{z}\right| j \circ\right)=J_{N}\left(2+\left\langle j\left|\hat{\sigma}_{N}^{z}\right| j\right\rangle\right) / h_{N}+O\left(J_{N}^{2}\right)
$$

Similarly,

$$
\left(j \bullet\left|\hat{\sigma}_{N+1}^{z}\right| j \bullet\right)=-J_{N}\left(2+\left\langle j\left|\hat{\sigma}_{N}^{z}\right| j\right\rangle\right) / h_{N}+O\left(J_{N}^{2}\right) .
$$

Induction hypothesis 2 for $H_{N}$ implies that $\left\{\left(j \circ\left|\hat{\sigma}_{N+1}^{z}\right| j \circ\right),\left(j \bullet\left|\hat{\sigma}_{N+1}^{z}\right| j \bullet\right)\right\}_{j=1}^{2^{N}}$ is a set of pairwise distinct numbers.

\section{Extensions of Theorem 2}

\section{C.1 Proof of Corollary 2}

Following the proof of Theorem 2 in Appendix $B$, it suffices to find a particular $\gamma \in \mathbb{R}^{3 N-1}$ (not necessarily $\gamma \in[-1,1]^{\times(3 N-1)}$ ) such that the spectrum of $H_{h}^{X X Z}(\gamma)$ has non-degenerate gaps. Let

$$
H_{N}^{\prime}:=\Lambda H_{N}+\sum_{j=1}^{N-1}\left(\hat{\sigma}_{j}^{x} \hat{\sigma}_{j+1}^{x}+\hat{\sigma}_{j}^{y} \hat{\sigma}_{j+1}^{y}\right),
$$

where $H_{N}$ is given by Eq. (48). Since the spectrum of $H_{N}$ has non-degenerate gaps (Lemma 7), that of $H_{N}^{\prime}$ also has non-degenerate gaps for sufficiently large $\Lambda>0$. 


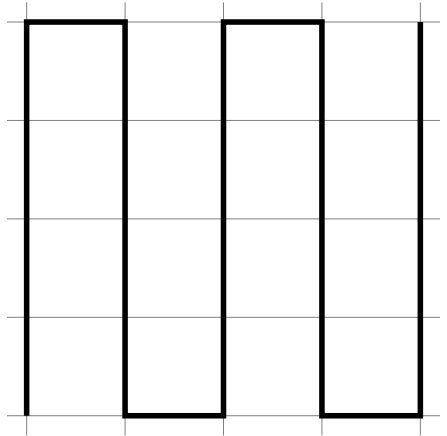

Figure 1: Embedding a spin chain (thick line) into a two-dimensional square lattice (grid) such that adjacent sites in the spin chain remain adjacent in the square lattice.

\section{C.2 Higher spatial dimensions}

To prove an analogue of Theorem 2 on a higher-dimensional lattice $L$, it suffices to construct a particular Hamiltonian on $L$ with nearest-neighbor interactions such that its spectrum has non-degenerate gaps. To this end, we simply embed the spin chain model (48) into L. Figure 1 illustrates the embedding for $L$ being a two-dimensional square lattice.

\section{C.3 Qudit systems}

To extend Theorem 2 to a qudit chain with $d \geq 3$, it suffices to prove an analogue of Lemma 7. Let $-1 \leq e_{1}<e_{2}<\cdots<e_{d} \leq 1$ be $d$ constants such that the spectrum of the diagonal matrix $\hat{X}:=\operatorname{diag}\left(e_{1}, e_{2}, \ldots, e_{d}\right)$ has non-degenerate gaps. Let

$$
\hat{Z}:=\bigoplus_{j=1}^{d / 2}\left(\begin{array}{ll}
0 & 1 \\
1 & 0
\end{array}\right), \quad \hat{Z}:=\bigoplus_{j=1}^{(d-1) / 2}\left(\begin{array}{ll}
0 & 1 \\
1 & 0
\end{array}\right) \oplus(0)
$$

if $d$ is even/odd, respectively, so that the expectation value of $\hat{Z}$ in any eigenstate of $\hat{X}$ is 0 . Let $\Lambda>1$ be a constant such that

$$
\Lambda>\max _{1 \leq j<k \leq d / 2} \frac{e_{2 j}-e_{2 j-1}+e_{2 k}-e_{2 k-1}}{\left|e_{2 j}-e_{2 j-1}-e_{2 k}+e_{2 k-1}\right|}
$$

Consider a chain of $N$ qudits. Let $\hat{X}_{j}, \hat{Z}_{j}$ be the $\hat{X}, \hat{Z}$ operators for the spin at position $j$. It is trivial to construct a Hamiltonian $H_{1}$ acting only on the first spin such that

1. The spectrum of $H_{1}$ has non-degenerate gaps.

2. $\left\{\left\langle j\left|\hat{Z}_{1}\right| j\right\rangle\right\}$ is a set of pairwise distinct numbers, where $\{|j\rangle\}_{j=0}^{d-1}$ are eigenstates of $H_{1}$.

Corollary 3. For any positive integer $N$, there exists $\left(h_{1}, J_{1}, h_{2}, J_{2}, \ldots, h_{N-1}, J_{N-1}\right) \in$ $\mathbb{R}^{2(N-1)}$ such that the spectrum of

$$
H_{N}=H_{1}+\sum_{j=1}^{N-1}\left(h_{j} \hat{X}_{j+1}+J_{j}\left(\Lambda+\hat{Z}_{j}\right) \hat{Z}_{j+1}\right)
$$

has non-degenerate gaps.

This corollary can be proved in almost the same way as Theorem 2 . 


\section{Proof of Theorem 3}

Lemma 8. Let $\{|j\rangle\}_{j=0}^{d-1}$ be an orthonormal basis of $\mathbb{C}^{d}$. For a Haar-random state $\left|\Psi^{\prime}\right\rangle$,

$$
-\underset{\Psi^{\prime}}{\mathbb{E}} \sum_{j=0}^{d-1} p_{j} \ln p_{j}=\sum_{k=2}^{d} \frac{1}{k}, \quad p_{j}:=\left|\left\langle j \mid \Psi^{\prime}\right\rangle\right|^{2} .
$$

Proof.

$$
\underset{\Psi^{\prime}}{\mathbb{E}} \sum_{j=0}^{d-1} p_{j} \ln p_{j}=d \underset{\Psi^{\prime}}{\mathbb{E}} p_{0} \ln p_{0}=d \int_{0}^{1} x f(x) \ln x \mathrm{~d} x=-\sum_{k=2}^{d} \frac{1}{k},
$$

where $f(x)$ is given by Eq. 23.

Let

$$
\sigma_{A}:=\lim _{\tau \rightarrow+\infty} \frac{1}{\tau} \int_{0}^{\tau} \rho_{A}(t) \mathrm{d} t
$$

be the infinite time average and $\sigma_{j}:=\operatorname{tr}_{A \backslash\{j\}} \sigma_{A}$ be the reduced density matrix of the spin at position $j \in A$. Using the concavity and subadditivity [2] of the von Neumann entropy,

$$
\lim _{\tau \rightarrow+\infty} \frac{1}{\tau} \int_{0}^{\tau} S\left(\rho_{A}(t)\right) \mathrm{d} t \leq S\left(\sigma_{A}\right) \leq \sum_{j \in A} S\left(\sigma_{j}\right)
$$

Let $x \doteq y$ denote that $x-y$ is infinitesimal. Recall that $H^{\mathrm{mbl}} \doteq H^{\text {loc }}$, which is diagonal in the computational basis and whose spectrum is non-degenerate. For a Haar-random product state $|\Psi\rangle=\bigotimes_{j=1}^{N}\left|\Psi_{j}\right\rangle$, it is not difficult to see that

$$
\sigma_{j} \doteq \operatorname{diag}\left(p_{j, 0}, p_{j, 1}, \ldots, p_{j, d-1}\right), \quad p_{j, k}:=\left|{ }_{j}\left\langle k \mid \Psi_{j}\right\rangle\right|^{2}
$$

where $\left\{|k\rangle_{j}\right\}_{k=0}^{d-1}$ is the computational basis for the spin at position $j$. Hence,

$$
\underset{\Psi}{\mathbb{E}} \sum_{j \in A} S\left(\sigma_{j}\right) \doteq-\sum_{j \in A} \underset{\Psi_{j}}{\mathbb{E}} \sum_{k=0}^{d-1} p_{j, k} \ln p_{j, k} .
$$

Theorem 3 follows by combining Lemma 8 and (67), 69).

\section{References}

[1] H. Abdul-Rahman, B. Nachtergaele, R. Sims, and G. Stolz. Entanglement dynamics of disordered quantum XY chains. Letters in Mathematical Physics, 106(5):649-674, 2016.

[2] H. Araki and E. H. Lieb. Entropy inequalities. Communications in Mathematical Physics, 18(2):160-170, 1970.

[3] K. M. R. Audenaert. A sharp continuity estimate for the von Neumann entropy. Journal of Physics A: Mathematical and Theoretical, 40(28):8127-8136, 2007. 
[4] J. H. Bardarson, F. Pollmann, and J. E. Moore. Unbounded growth of entanglement in models of many-body localization. Physical Review Letters, 109(1):017202, 2012.

[5] M. Fannes. A continuity property of the entropy density for spin lattice systems. Communications in Mathematical Physics, 31(4):291-294, 1973.

[6] T. Farrelly, F. G. S. L. Brandão, and M. Cramer. Thermalization and return to equilibrium on finite quantum lattice systems. Physical Review Letters, 118(14):140601, 2017.

[7] Y. Huang and A. W. Harrow. Instability of localization in translation-invariant systems. arXiv:1907.13392, 2019.

[8] D. A. Huse, R. Nandkishore, and V. Oganesyan. Phenomenology of fully many-bodylocalized systems. Physical Review B, 90(17):174202, 2014.

[9] J. Z. Imbrie. On many-body localization for quantum spin chains. Journal of Statistical Physics, 163(5):998-1048, 2016.

[10] E. H. Lieb and M. B. Ruskai. Proof of the strong subadditivity of quantum-mechanical entropy. Journal of Mathematical Physics, 14(12):1938-1941, 1973.

[11] N. Linden, S. Popescu, A. J. Short, and A. Winter. Quantum mechanical evolution towards thermal equilibrium. Physical Review E, 79(6):061103, 2009.

[12] A. Nanduri, H. Kim, and D. A. Huse. Entanglement spreading in a many-body localized system. Physical Review B, 90(6):064201, 2014.

[13] P. Reimann. Foundation of statistical mechanics under experimentally realistic conditions. Physical Review Letters, 101(19):190403, 2008.

[14] P. Reimann and M. Kastner. Equilibration of isolated macroscopic quantum systems. New Journal of Physics, 14(4):043020, 2012.

[15] M. Serbyn, Z. Papić, and D. A. Abanin. Universal slow growth of entanglement in interacting strongly disordered systems. Physical Review Letters, 110(26):260601, 2013.

[16] A. J. Short. Equilibration of quantum systems and subsystems. New Journal of Physics, 13(5):053009, 2011.

[17] A. J. Short and T. C. Farrelly. Quantum equilibration in finite time. New Journal of Physics, 14(1):013063, 2012.

[18] R. Vosk and E. Altman. Many-body localization in one dimension as a dynamical renormalization group fixed point. Physical Review Letters, 110(6):067204, 2013.

[19] H. Wilming, M. Goihl, I. Roth, and J. Eisert. Entanglement-ergodic quantum systems equilibrate exponentially well. Physical Review Letters, 123(20):200604, 2019.

[20] M. Znidaric, T. Prosen, and P. Prelovsek. Many-body localization in the Heisenberg XXZ magnet in a random field. Physical Review B, 77(6):064426, 2008. 